\title{
Monofractal and Multifractal Approaches to Complex Biomedical Signals
}

\author{
H. E. Stanley, ${ }^{*}$ L. A. N. Amaral, ${ }^{* \dagger}$ A. L. Goldberger, ${ }^{\dagger}$ \\ S. Havlin, ${ }^{* \ddagger}$ P. Ch. Ivanov ${ }^{* \dagger}$ and C.-K. Peng ${ }^{\dagger}$ \\ * Center for Polymer Studies and Department of Physics \\ Boston University, Boston, MA 02215, USA \\ $\dagger_{\text {Harvard Medical School, Beth Israel Deaconess Medical Center }}$ \\ Boston, MA 02015 USA \\ $\ddagger$ Gonda Goldschmid Center and Department of Physics \\ Bar-Ilan University, Ramat Gan, Israel
}

\begin{abstract}
Even under healthy, basal conditions, physiologic systems show erratic fluctuations resembling those found in dynamical systems driven away from an equilibrium state. Do such "nonequilibrium" fluctuations simply reflect the fact that physiologic systems are being constantly perturbed by external and intrinsic uncorrelated noise? Or, do these fluctuations actually contain "hidden" information about the underlying nonequilibrium control mechanisms? We report some recent attempts to understand the dynamics of complex physiologic fluctuations by adapting and extending concepts and methods developed very recently in statistical physics. Specifically, we focus on interbeat interval variability as an important quantity to help elucidate possibly nonhomeostatic physiologic variability because (i) the heart rate is under direct neuroautonomic control, (ii) interbeat interval variability is readily measured by noninvasive means, and (iii) analysis of these heart rate dynamics may provide important practical diagnostic and prognostic information not obtainable with current approaches. The analytic tools we discuss may be used on a wider range of physiologic signals. We first review recent progress using two analysis methods-detrended fluctuation analysis and wavelets-appropriate for quantifying monofractal structures. We then describe very recent work that quantifies multifractal features of interbeat interval series, and the discovery that the multifractal structure of healthy subjects is different from that of diseased subjects. We also discuss the application of fractal scaling analysis to the dynamics of heartbeat regulation, and report the recent finding that the scaling exponent $\alpha$ is smaller during sleep periods compared to wake periods.
\end{abstract}

\section{INTRODUCTION}

A central task of statistical physics is to deal with macroscopic phenomena that result from microscopic interactions among many individual components [1]. This problem, which is at the root of many of the contributions to this conference, is a

CP502, Stochastic and Chaotic Dynamics in the Lakes: STOCHAOS,

edited by D. S. Broomhead, E. A. Luchinskaya, P.V. E. McClintock, and T. Mullin

(C) 2000 American Institute of Physics 1-56396-915-7/00/\$17.00

133 
problem on which much progress has been made in the last third of this century. In particular, physiologic systems under neuroautonomic regulation $[2,3]$, such as heart rate regulation, are good candidates for such an approach, since (i) the systems often include multiple components, thus leading to many degrees of freedom, and (ii) the systems usually are driven by competing forces, e.g., parasympathetic versus sympathetic stimuli. Therefore, it seems reasonable to consider the possibility that dynamical systems under neuroautonomic regulation may exhibit temporal structures that are similar, under certain conditions, to those found in physical systems. Indeed, new conceptual frameworks and corresponding methodologies are being developed in order to deal with three particularly vexing features of physiologic time series:

(i) Nonstationarity. Traditional methods of statistical analysis assume that the statistical properties of a signal are the same throughout the signal [4]. This is not true for many signals of interest in physiology-e.g., the statistical properties of the heart rate change when a subject rises to a standing position. Such nonstationarity problems arise in other contexts in the discipline of statistical physics, and novel techniques such as detrended fluctuation analysis (DFA) [5] and wavelets [6-8] have been successfully developed to study nonstationary signals. Hence we are exploring the degree to which the solutions found in statistical physics can be usefully applied to physiologic signals.

(ii) Nonlinearity. Traditional methods of analysis also assume that to a large degree the system can be viewed as linear, so that departures from linearity can be treated perturbatively. This is not true for most physiologic systems, which are intrinsically nonlinear. A salient feature of nonlinear systems is that their components interact with each other, and therefore their outputs are not proportional to the strength of the inputs. The field of statistical physics has in the past 10 years focused on nonlinear systems, and has developed a conceptual framework within which a wide range of nonlinear phenomena can be usefully treated. Hence we are seeking to uncover which of these methodologies can be usefully applied and carefully adapted to data. In particular, multifractal methods $[9,10]$ offer a new and potentially promising avenue for quantifying features of a range of physiological signals that differ in health and disease.

(iii) Nonequilibrium phenomena. From the time of Claude Bernard [11] and Walter Cannon [12], it has been assumed that physiologic systems possess feedback and control mechanisms that serve to restore an equilibrium-like state when a system is perturbed away from some set point. Recent research, however, has shown that physiologic systems are inherently out-of-equilibrium systems [13]. Nonequilibrium statistical mechanics has made advances in recent years that have yet to be applied in the physiologic domain.

The statistical methods we are developing are particularly attractive for the analysis of heart rate time series because they can be reliably applied to complex signals from stochastic, deterministic, or mixed systems. Further, these techniques are specifically designed to cope with the output of highly nonstationary processes. As such, these methods complement approaches derived from the analysis of 
deterministic systems which may be less appropriate for nonstationary data [14-16].

\section{INFORMATION IN NONSTATIONARITY PHYSIOLOGIC SIGNALS}

A major problem in contemporary physiology is the presence of nonstationarity in time series. The signals obtained under constantly varying conditions raise serious challenges to both technical and theoretical aspects of time series analyses.

Representative examples of complex dynamical behavior under physiologic and pathologic conditions are cardiac interbeat time series - the output of a spatially and temporally integrated neuroautonomic control system. These time series show erratic fluctuations and "patchiness." These fluctuations are usually ignored in conventional medical studies that focus on averaged quantities. In fact, these fluctuations are often labeled as "noise" to distinguish them from the true "signal" of interest. In the conventional approach it is assumed that there is no meaningful structure in apparent noise and, therefore, one does not expect to gain any understanding about the underlying system through the study of these nonequilibrium fluctuations. However, by adapting and extending methods developed in modern statistical physics and nonlinear mathematics, we have recently found that the physiologic fluctuations exhibit unexpected hidden scaling structure. Furthermore, these patterns change with pathological perturbations. These findings raise the possibility that understanding the origin of such temporal structures and their alterations may (i) elucidate certain basic features of heart rate control mechanisms, and (ii) have practical value in clinical monitoring.

\section{LIMITATIONS OF TRADITIONAL TECHNIQUES}

\section{Averages, Standard Deviations and Distribution Functions}

A technique widely used to analyze time series is the study of the moments of the distribution of measured values. Visual inspection makes clear the existence of differences in the dynamics generating signals from a normal individual and from a subject with congestive heart failure. However, the signals might have the same averages and standard deviations. Hence additional methods are required if these two signals are to be distinguished.

\section{The Power Spectrum of Nonstationary Signals}

A quantity widely used to measure correlations in a time series is the power spectrum, which measures the relative frequency content of a signal. A power spectrum calculation assumes that the signal studied is stationary, and when applied to nonstationary time series can lead to misleading results. To illustrate this 
point, we analyze two artificial signals: one is stationary-two different frequencies are present at all times. The other is nonstationary - one frequency is present in the first half of the signal and another frequency in the other half. The calculation of the power spectrum for these signals leads to almost identical results! Similarly, the presence of linear or higher order polynomial trends can mask the frequency content of a signal. Since the power spectrum is incapable of distinguishing between these types of behavior, it must not be used as the only form of analysis for nonstationary signals.

\section{MONOFRACTAL ANALYSIS: DETECTING AND QUANTIFYING LONG-RANGE CORRELATIONS}

To quantitatively describe noisy cardiac signals is not an easy task. Techniques for analysis must be selected carefully in order to extract robust features hidden in these complex fluctuations. We have developed several complementary algorithms in the last few years for this purpose. We will first discuss some interesting results obtained by applying these new methods.

\section{Measurement of Long-Range Correlations in Physiologic Interbeat Interval Dynamics}

An important question is whether the "heterogeneous" structure of physiologic time series arises trivially from external and intrinsic perturbations which push the system away from a homeastatic set point. An important alternative hypothesis is that the fluctuations are, at least in part, due to the underlying dynamics of the system. The key problem is how to decompose subtle fluctuations (due to intrinsic physiologic control) from other nonstationary trends associated with external stimuli.

To this end, our multidisciplinary team introduced the detrended fluctuation analysis (DFA) method [5]. The advantages of DFA over conventional methods are that it permits the detection of long-range correlations embedded in a seemingly nonstationary time series and also avoids the spurious detection of apparent longrange correlations that are an artifact of nonstationarity. The DFA method has been tested on control time series that consist of long-range correlations with superposition of a nonstationary external trend. It has also been successfully applied to detect long-range correlations in highly heterogeneous DNA sequences [5,17-19]. Of note is a recent independent review of fractal fluctuation analysis methods which determined that DFA was one of the most robust methods [20].

Briefly, a moving window of size $n$ is used to study how the fluctuation $F(n)$ grows with $n$ for the interbeat interval time series. The slope of the line relating $\log F(n)$ to $\log n$ determines the scaling exponent (self-similarity parameter) $\alpha$. The full computational details of the DFA method are described in Refs. [5,17]. 


\section{Alteration of Correlation Properties in Pathologic States}

Assessing correlations under pathologic conditions is likely to be particularly informative for patients with congestive heart failure since these individuals have abnormalities in both the sympathetic and parasympathetic control mechanisms [21] that regulate beat-to-beat variability. Previous studies have demonstrated marked changes in short-range heart rate dynamics in heart failure compared to healthy function, including the emergence of intermittent relatively low frequency ( $\sim 1$ cycle/minute) heart rate oscillations associated with the well-recognized syndrome of periodic (Cheyne-Stokes) respiration, an abnormal breathing pattern often associated with low cardiac output [21]. Of note is the fact that patients with congestive heart failure are at very high risk for sudden cardiac death.

To study the alteration of long-range correlations with pathology, we analyzed cardiac interbeat data from three different groups of subjects: (i) 29 adults (17 male and 12 female) without clinical evidence of heart disease (age range: 20-64 years, mean 41), (ii) 10 subjects with fatal or near-fatal sudden cardiac death syndrome (age range: $35-82$ years) and (iii) 15 adults with severe heart failure (age range: 22-71 years; mean 56). Data from each subject contains approximately 24 hours of ECG recording encompassing $\sim 10^{5}$ heartbeats.

For the normal control group, we observed $\alpha=1.00 \pm 0.10$ (mean value \pm S.D.). These results indicate that healthy heart rate fluctuations exhibit long-range powerlaw (fractal) correlation behavior over three decades, similar to that observed in many dynamical systems far from equilibrium $[22,23]$. Furthermore, both pathologic groups show significant deviation of the long-range correlations exponent $\alpha$ from the normal value, $\alpha=1$. For the group of heart failure subjects, we find that $\alpha=1.24 \pm 0.22$, while for the group of sudden cardiac death syndrome subjects, we find that $\alpha=1.22 \pm 0.25$. This result is consistent with our previous finding $[24,25]$ that there is a significant difference in the long-range scaling behavior between healthy and diseased states. Of particular note, we obtained similar results when we divided the time series into three consecutive subsets (of $\sim 8$ hours each) and repeated the above analysis [26]. Therefore our findings are not simply attributable to different levels of daily activities. Our results have been independently verified in Ref. [27].

\section{Differences in Correlation between Sleep and Awake Stages}

It is known that circadian rhythms are associated with periodic changes in key physiological processes $[2,28,29]$. Here, we review a recent study [30] asking if there are characteristic differences in the scaling behavior between sleep and wake cardiac dynamics. Typically the differences in the cardiac dynamics during sleep and wake phases are reflected in the average (higher in sleep) and standard deviation (lower in sleep) of the interbeat intervals [29]. Such differences can be easily observed in plots of the interbeat intervals recorded from subjects during sleep and wake 
periods. The hypothesis is that sleep and wake changes in cardiac control may occur on all time scales and thus could lead to systematic changes in the scaling properties of the heartbeat dynamics. Elucidating the nature of these sleep-wake rhythms could lead to a better understanding of the neuroautonomic mechanisms of cardiac regulation.

Ivanov et al. [30] analyzed 30 datasets - each with $24 \mathrm{~h}$ of interbeat intervalsfrom 18 healthy subjects and 12 patients with congestive heart failure [31]. The nocturnal and diurnal fractions of the dataset of each subject correspond to the $6 \mathrm{~h}$ ( $n \approx 22,000$ beats) from midnight to $6 \mathrm{am}$ and and from noon to $6 \mathrm{pm}$.

The detrended fluctuation analysis (DFA) method [5] was applied to quantify long-range correlations embedded in the nonstationary heartbeat time series. Reference [30] reported that at scales above $\approx \operatorname{lmin}(n>60)$ the data during wake hours display long-range correlations over two decades with average exponents $\alpha_{W} \approx 1.05$ for the healthy group and $\alpha_{W} \approx 1.2$ for the heart failure patients. For the sleep data, Ref. [30] found a crossover at scale $n \approx 60$ beats, followed by a scaling regime extending over two decades characterized by a smaller exponent: $\alpha_{S} \approx 0.85$ for the healthy group and $\alpha_{S} \approx 0.95$ for the heart failure group. Although the values of the sleep and wake exponents vary from subject to subject, Ref. [30] found that for all individuals studied, the heartbeat dynamics during sleep are characterized by a smaller exponent.

As a control, an identical analysis was performed on two surrogate data sets obtained by reshuffling and integrating the increments in the interbeat intervals of the sleep and wake records from the same healthy subject. Both surrogate sets display uncorrelated random walk fluctuations with a scaling exponent of 1.5 (Brownian noise). A scaling exponent larger than 1.5 would indicate persistent correlated behavior, while exponents with values smaller then 1.5 characterize anticorrelations (a perfectly anticorrelated signal would have an exponent close to zero). Our results therefore suggest that the interbeat fluctuations during sleep and wake phases are long-range anticorrelated but with a significantly greater degree of anticorrelation (smaller exponent) during sleep. These results suggest that the observed sleep-wake scaling differences are due to intrinsic changes in the cardiac control mechanisms.

The robustness of the above results was tested by analyzing 17 datasets from 6 cosmonauts during long-term orbital flight on the Mir space station [32]. Each dataset contains continuous periods of $6 \mathrm{~h}$ data under both sleep and wake conditions. Reference [30] found that for all cosmonauts the heartbeat fluctuations exhibit anticorrelated behavior with average scaling exponents consistent with those found for the healthy terrestrial group: $\alpha_{W} \approx 1.04$ for the wake phase and $\alpha_{S} \approx 0.82$ for the sleep phase. This sleep-wake scaling difference is observed not only for the group averaged exponents but for each individual cosmonaut dataset. Moreover, the scaling differences are persistent in time, since records of the same cosmonaut taken on different days (ranging from the 3rd to the 158th day in orbit), exhibit a higher degree of anticorrelation during sleep.

The finding of stronger heartbeat anticorrelations during sleep is of interest from a physiological viewpoint, since it may motivate new modeling approaches and 
supports a reassessment of the sleep phase as a surprisingly active dynamical state. Perhaps the "restorative" functions of sleep may relate to an increased reflexive-type responsiveness of neuroautonomic control, not just at one characteristic frequency, but over a broad range of time scales.

Recent work by Ivanov et al. [13] indicates that key statistical characteristics of the healthy cardiac dynamics can be successfully reproduced by a stochastic nonlinear feedback mechanism. The present observation of sleep-wake scaling differences poses a new challenge to such modeling approaches, which could require considering reciprocity in the activity of the sympathetic and parasympathetic branches of the autonomic nervous system during sleep and wake phases, as well as different correlation times of the sympathetic and parasympathetic impulses.

\section{WAVELET ANALYSIS OF HEARTBEAT INTERVALS}

Time series of beat-to-beat heart rate intervals obtained from digitized electrocardiograms are known to be nonstationary and exhibit extremely complex behavior [33-35]. A typical feature of these signals is the presence of "patchy" patterns which change over time. Heterogeneous properties may be even more strongly expressed in certain cases of abnormal heart activity. Traditional approaches - such as the power spectrum and correlation analysis [36,37] — are not suited for such nonstationary sequences, and do not carry information stored in the Fourier phases.

To address these problems, we present an alternative method- "cumulative variation magnitude analysis" [38] — to study the subtle structure of physiological time series. This method comprises sequential application of a set of algorithms based on wavelet and Hilbert transform analysis. First, we apply the wavelet transform, because it does not require stationarity and preserves the Fourier phase information. The wavelet transform is sometimes called a "mathematical microscope" because it allows one to study properties of the signal on any chosen scale $a$. However, a wavelet with too large a value of scale $a$ (low frequency) will filter out almost the entire frequency content of the time series, thus losing information about the intrinsic dynamics of the system. We focus our "microscope" on scale $a=8$ beats which smoothes locally very high-frequency variations and best probes patterns of specific duration $\left(\approx \frac{1}{2}-1 \mathrm{~min}\right)$. The wavelet transform is attractive because it can eliminate local polynomial behavior in the nonstationary signal by an appropriate choice of the analyzing wavelet $\psi$.

The wavelet transform is thus a cumulative measure of the variations in the heart rate signal over a region proportional to the wavelet scale, so study of the behavior of the wavelet values can reveal intrinsic properties of the dynamics masked by nonstationarity.

The second step of the cumulative variation magnitude analysis is to extract the instantaneous variation amplitudes of the wavelet-filtered signal by means of an analytic signal approach $[36,39]$ which also does not require stationarity. Let $s(t)$ represent an arbitrary signal. The analytic signal, a complex function of time, is 
defined by $S(t)=s(t)+i \tilde{s}(t)=A(t) \epsilon^{i \dot{\phi}(t)}$, where $\tilde{s}(t)$ is the Hilbert transform [40] of $s(t)$. The instantaneous magnitude $A(t)$ and the instantaneous phase of the signal $\phi(t)$ are defined as $A(t) \equiv \sqrt{s^{2}(t)+\tilde{s}^{2}(t)}$ and $\phi(t) \equiv \tan ^{-1}(\tilde{s}(t) / s(t))$.

We study the distribution of the amplitudes of the beat-to-beat variations for a group of healthy subjects $(N=18 ; 5$ male, 13 female; age: $20-50$, mean - 34) and a group of subjects [31] with obstructive sleep apnea [41] $(N=16$ males; age: $32-56$, mean - 43). We begin by considering night phase $(12 \mathrm{pm}-$ $6 \mathrm{am}$ ) records of interbeat intervals $\left(\approx 10^{4}\right.$ beats $)$ for both groups to minimize nonstationarity due to changes in the level of activity. Inspection of the distribution functions of the amplitudes of the cumulative variations reveals marked differences between individuals. These discrepancies are not surprising given the underlying physiological differences among healthy subjects. To test the hypothesis that there is a hidden, possibly universal structure to these heterogeneous time series, we rescale the distributions and find for all healthy subjects that the data conform to a single scaled plot ("data collapse"). We are able to describe the distributions using a single curve, indicating a robust, consistent scaling mechanism for the healthy nonequilibrium dynamics. Such behavior is reminiscent of a wide class of well-studied physical systems with universal scaling properties $[1,9,3]$. In contrast, the subjects with sleep apnea show individual probability distributions which fail to collapse.

The absence of data collapse demonstrates deviation from the normal heart behavior. We note that direct analysis of interbeat interval histograms does not lead to data collapse or separation between the healthy and apnea group [42]. Moreover, we find that the direct application of the Hilbert transform yielding the probability distribution of the instantaneous amplitudes of the original signal does not clearly distinguish healthy from abnormal cardiac dynamics. Hence the crucial feature of the wavelet transform is that it extracts dynamical properties hidden in the cumulative variations. We observe for the healthy group good data collapse with a stable scaling form for wavelet scales $a=2$ up to $a=32$. However, for very small scales $(a=1,2)$ the average of the rescaled distributions of the apnea group is indistinguishable from the average of the rescaled distributions of the healthy group. Hence very high frequencies are equally present in the signals from both groups. Our analysis yields the most robust results when $a$ is tuned to probe the collective properties of patterns with duration of $\approx \frac{1}{2}-1 \mathrm{~min}$ in the time series $(a=8,10)$. The subtle difference in the distributions of the cumulative variations between day and night phases is also best seen for this scale range [43].

It has been hypothesized [42] that even if the interbeat variations are different (e.g. smaller) during illness, the pattern of heart rate variability might be otherwise very similar to that during health, so that the interbeat variations for normal and abnormal cardiac dynamics, once normalized, would have the same distribution. Our study clearly rejects this hypothesis, showing the presence of scaling in the distributions of the variation amplitudes for the healthy and a breakdown of this scaling for abnormal dynamics. Moreover, the stability of this scaling form indicates 
that the underlying dynamical mechanisms regulating the healthy heart beat have similar statistical properties on different time scales. Such statistical self-similarity is an important characteristic of fractal objects. The wavelet decomposition of beat-to-beat heart rate signals can be used to provide a visual representation of this fractal structure. The wavelet transform, with its ability to remove local trends and to extract interbeat variations on different time scales, enables us to identify self-similar patterns (arches) in these variations even when the signals change as a result of background interference. Data from sick heart lack these patterns.

The study of Ivanov et al. [38] uncovers a previously unknown nonlinear feature of healthy heart rate fluctuations. Prior reports of universal properties of the normal heart beat and other physiological signals relate to long-range correlations and power law scaling. However, these properties, detected by Fourier and fluctuation analysis techniques, ignore information related to the phase interactions of component modes [8]. The nonlinear interaction of these modes accounts for the patchy, non-homogeneous appearance of the heartbeat time series and appears to be related to the recently reported multifractal properties of the heartbeat dynamics [44] . This finding suggests that for healthy individuals, there may be a common structure to this nonlinear phase interaction.

\section{MULTIFRACTAL ANALYSIS: APPLICATION TO PHYSIOLOGIC SIGNALS}

The DFA method can measure only one exponent characterizing a given signal. This fact implies that the method is more appropriate for the study of monofractal signals. Monofractals are homogeneous in the sense that they have the same scaling properties, characterized by a single scaling exponent [9,45-49]. On the other hand, multifractal signals or objects require an infinite number of indices to characterize their scaling properties. Multifractals can be decomposed into many-possibly infinitely many - sub-sets characterized by different exponents $h$. The singularity spectrum, $D(h)$, quantifies the fractal dimension of the sub-set characterized by the exponent $h$. Thus, multifractal signals are intrinsically more complex, and inhomogeneous, than monofractals.

Multifractal structures have been uncovered in a number of classical physical problems such as voltage drops across a random resistor network [50], spatial distribution of the dissipation field of fully developed turbulence [51], viscous fingering $[52,53]$, and diffusion limited aggregation $[10,54]$. However, in physics and other applied sciences, fractals appear not only as singular objects (measures) but also as singular functions generated by dynamical systems. There have been only a few attempts to extend the concept of multifractality to singular functions: for velocity in turbulence [55-57] and for rough surfaces [58].

Physiologic signals are generated by complex self-regulating systems that process inputs with a broad range of characteristics. Monofractal signals are homogeneous and have "linear" properties. Many physiologic time series-such as interbeat 
interval sequences-are in fact inhomogeneous, suggesting that different parts of the signal have different scaling properties. In addition, there is evidence that heartbeat dynamics exhibits nonlinear properties $[30,38,43]$. Up to now, robust demonstration of multifractality for nonstationary time series has been hampered by problems related to a drastic bias in the estimate of the singularity spectrum due to diverging negative moments. Moreover, the classical approaches based on the box-counting technique and structure function formalism fail when a fractal function is composed of a multifractal singular part embedded in regular polynomial behavior.

We recently adopted this new methodology to human heartbeat interval series obtained from electrocardiogram records [44]. Our initial findings include several encouraging results:

- (i) The heart rate of healthy humans is a multifractal with nonzero fractal dimension for sub-sets characterized by singularity exponents in the interval -0.1 to 0.5 .

- (ii) Records for patients with a nearly terminal pathology, congestive heart failure, show a significant loss of multifractal complexity displaying a smaller range of values of $h$.

¿From a physiologic perspective, the detection of rubust multifractal scaling in heart rate dynamics is of interest for a number of reasons. First, previous analyses have focused only on the quantification of a single scaling exponent (monofractal) behavior to account for the apparently $1 / f$ spectrum of healthy interbeat intervals over a wide range of time scales. We show for the first time that the healthy heartbeat is even more complex than previously suspected, requiring multifractal scaling with multiple exponents for its characterization. Second, our analysis indicates that the observed multifractality is related to nonlinear features of the healthy heartbeat dynamics, which are encoded in the Fourier phases $[38,44]$. Third, we find a loss of multifractal complexity in a major pathologic condition - namely congestive heart failure - suggesting possible bedside applications. Fourth, our results are notable because they pose a challenge to ongoing efforts to develop realistic models of heart rate control and other processes under neuroautonomic regulation. There is currently no precedent in physiology to account for such complex behavior which in physical systems has been connected with turbulence and related multiscale phenomena. Our findings raise the intriguing possibility that the control mechanisms regulating the heartbeat interact as part of a coupled cascade of feedback loops in a system operating far from equilibrium.

\section{ACKNOWLEDGEMENTS}

I conclude by thanking those under whose tutelage I learned what little I understand of this subject. In addition to those who consented to join me in co-authoring 
this brief report, these include the students, postdocs, and faculty visitors to our research group with whom I have enjoyed the pleasure of scientific collaboration. Those whose research provided the basis of this short report include, in addition to the co-authors: S. V. Buldyrev, J. M. Hausdorff, K. K. L. Ho, H. V. Huikuri, T. H. Mäkikallio, J. Mietus, M. G. Rosenblum, M. Simons, G. M. Viswanathan, and Z. Struzik. This research was supported by a grant from the National Institutes of Health/National Center for Research Resources (P41 RR13622).

\section{REFERENCES}

1. H. E. Stanley, Rev. Mod. Phys. 71, S358 (1999).

2. R. M. Berne and M. N. Levy, Cardiovascular Physiology, 6th Edition (C. V. Mosby, St. Louis, 1996).

3. J. B. Bassingthwaighte, L. S. Liebovitch, and B. J. West, Fractal Physiology (Oxford Univ. Press, New York, 1994).

4. W. Feller, An Introduction to Probability Theory and Its Applications, Vol. 1, Third edition (J. Wiley \& Sons, New York, 1968).

5. C.-K. Peng, S. V. Buldyrev, S. Havlin, M. Simons, H. E. Stanley, and A. L. Goldberger, Phys. Rev. E 49, 1691 (1994).

6. I. Daubechies, Ten Lectures on Wavelets (SIAM, Philadelphia, 1992).

7. A. Aldroubi and M. Unser, eds., Wavelets in Medicine and Biology (CRC Press, Boca Raton, 1996).

8. P. Ch. Ivanov, A. L. Goldberger, S. Havlin, C.-K. Peng, M. G. Rosenblum, and H. E. Stanley, in Wavelets in Physics, edited by J. van den Berg (Cambridge University Press, Cambridge, 1999).

9. T. Vicsek, Fractal Growth Phenomena, Second Edition (World Scientific, Singapore, 1992).

10. H. E. Stanley and P. Meakin, Nature 335, 405 (1988).

11. C. Bernard, Les Phénoménes de la Vie (Paris, 1878).

12. W. B. Cannon, Physiol. Rev. 9, 399 (1929).

13. P. Ch. Ivanov, L. A. N. Amaral, A. L. Goldberger and H. E. Stanley, Europhys. Lett. 43, 363 (1998).

14. J. Theiler, S. Eubank, A. Longtin, B. Galdrikian, and J. D. Farmer, Physica D 58, 77 (1992).

15. A. R. Osborne and A. Provenzale, Physica D 35, 357 (1989).

16. P. E. Rapp, Integr. Physiol. Behav. Sci. 29, 311 (1994).

17. S. V. Buldyrev, A. L. Goldberger, S. Havlin, R. N. Mantegna, M. E. Matsa, C.-K. Peng, M. Simons, and H. E. Stanley, Phys. Rev. E 51, 5084 (1995).

18. S. V. Buldyrev, A. L. Goldberger, S. Havlin, C.-K. Peng, H. E. Stanley, and M. Simons, Biophys. J. 65, 2673 (1993).

19. S. M. Ossadnik, S. V. Buldyrev, A. L. Goldberger, S. Havlin, R. N. Mantegna, C.-K. Peng, M. Simons, and H. E. Stanley, Biophys. J. 67, 64 (1994).

20. M. S. Taqqu, V. Teverovksy, and W. Willinger, Fractals 3, 785 (1996). 
21. A. L. Goldberger, D. R. Rigney, J. Mietus, E. M. Antman, and M. Greenwald, Experientia 44, 983 (1988).

22. F. Mallamace and H. E. Stanley, eds., Physics of Complex Systems: Proc. Enrico Fermi School on Physics, Course CXXXIV (IOS Press, Amsterdam, 1997).

23. P. Meakin, Fractals, Scaling and Growth Far from Equilibrium (Cambridge University Press, Cambridge, 1997).

24. C.-K. Peng, J. Mietus, J. M. Hausdorff, S. Havlin, H. E. Stanley, and A. L. Goldberger, Phys. Rev. Lett. 70, 1343 (1993).

25. S. Havlin, S. V. Buldyrev, A. L. Goldberger, P. Ch. Ivanov, C.-K. Peng, H. E. Stanley and G. M. Viswanathan, in The Physics of Complex Systems [Proceedings of the International School of Physics "Enrico Fermi," Course CXXXIV], edited by F. Mallamace and H. E. Stanley (IOS Press, Amsterdam, 1997), pp. 445-472.

26. C.-K. Peng, S. Havlin, H. E. Stanley, and A. L. Goldberger, Chaos 5, 82 (1995).

27. R. G. Turcott and M. C. Teich, Ann. of Biomed. Eng. 24, 269 (1996).

28. M. Malik and A. J. Camm, eds., Heart Rate Variability (Futura, Armonk NY, 1995).

29. H. Moelgaard, K. E. Soerensen, and P. Bjerregaard, Am. J. Cardiol. 68, 777 (1991).

30. P. Ch. Ivanov, A. Bunde, L. A. N. Amaral, S. Havlin, J. Fritsch-Yelle, R. M. Baevsky, H. E. Stanley, and A. L. Goldberger, Europhys. Lett. (in press).

31. MIT-BIH Polysomnographic Database CD-ROM, second edition (MIT-BIH Database Distribution, Cambridge, 1992). The database now includes 18 healthy subjects (13 female and 5 male, with ages between 20 and 50 , average 34.3 years), and 12 congestive heart failure subjects ( 3 female and 9 male, with ages between 22 and 71 , average 60.8 year) in sinus rhythm.

32. A. L. Goldberger, M. W. Bungo, R. M. Baevsky, B. S. Bennett, D. R. Rigney, J. E. Mietus, G. A. Nikulina, and J. B. Charles, Am. Heart J. 128, 202 (1994).

33. R. I. Kitney, D. Linkens, A. C. Selman and A. H. McDonald, Automedica 4, 141 (1982).

34. A. L. Goldberger, D. R. Rigney, J. Mietus, E. M. Antman and S. Greenwald, Experientia 44, 983 (1988).

35. A. L. Goldberger, Lancet 347, 1312 (1996).

36. D. Panter, Modulation, Noise and Spectral Analysis (McGraw-Hill, New York, 1965).

37. S. Akselrod, D. Gordon, F. A. Ubel, D. C. Shannon, A. C. Barger and R. J. Cohen, Science 213, 220 (1981).

38. P. Ch. Ivanov, M. G. Rosenblum, C.-K. Peng, J. Mietus, S. Havlin, H. E. Stanley, and A. L. Goldberger, Nature 383, 323 (1996).

39. L. A. Vainshtein and D. E. Vakman, Separation of Frequencies in the Theory of Oscillations and Waves (Nauka, Moscow, 1983).

40. D. Gabor, J. Inst. Elect. Engrs. 93, 429 (1946).

41. C. Guilleminault, S. Connolly, R. Winkle, K. Melvin, A. Tilkian: Lancet 1, 126 (1984).

42. A. A. Aghili et al., Phys. Rev. Lett. 74, 1254 (1995).

43. P. Ch. Ivanov, M. G. Rosenblum, C-K Peng, J. Mietus, S. Havlin, H. E. Stanley, and A. L. Goldberger, Physica A [Proc. Bar-Ilan Conf] 249, 587 (1998).

44. P. Ch. Ivanov, L. A. N. Amaral, A. L. Golberger, S. Havlin, M. G. Rosenblum, Z. Struzik and H. E. Stanley, Nature 399, 461 (1999). 
45. A. Bunde and S. Havlin, eds., Fractals in Science (Springer-Verlag, Berlin, 1994),

46. A. Bunde and S. Havlin, eds., Fractals and Disordered Systems, 2nd Edition (Springer-Verlag, Berlin, 1996).

47. A.-L. Barabási and H. E. Stanley, Fractal Concepts in Surface Growth (Cambridge University Press, Cambridge, 1995).

48. H. Takayasu, Fractals in the Physical Sciences (Manchester University Press, Manchester UK, 1990).

49. H. E. Stanley, Nature 378, 554 (1995).

50. L. de Arcangelis, S. Redner, and A. Coniglio, Phys. Rev. B 31, 4725 (1985).

51. U. Frisch, Turbulence (Cambridge University Press, Cambridge UK, 1995).

52. J. Nittmann, G. Daccord, and H. E. Stanley, Nature 314, 141 (1985).

53. J. Nittmann, and H. E. Stanley, Nature 321, 663 (1986).

54. P. Meakin, H. E. Stanley, A. Coniglio, and T. A. Witten, Phys. Rev. A 32, 2364 (1985).

55. J. F. Muzy, E. Bacry, and A. Arneodo, Phys. Rev. Lett. 67, 3515 (1991).

56. C. Meneveau and K. R. Sreenivasan, Phys. Rev. Lett. 59, 1424 (1987).

57. J. F. Muzy, E. Bacry, and A. Arneodo, Phys. Rev. E 47, 875 (1993).

58. A.-L. Barabási, P. Szépfalusy, and T. Vicsek, Physica A 178, 17 (1991). 